\title{
Understanding the Cost-Effectiveness of Hearing Aids and Surgery for the Treatment of Otosclerosis
}

\author{
Danielle M. Gillard ${ }^{1}$ [D . Jeffrey D. Sharon ${ }^{1}$
}

Accepted: 30 October 2021 / Published online: 4 January 2022

(c) The Author(s) 2022

\begin{abstract}
Purpose of Review To summarize and critically review recent literature on the relative cost-effectiveness of hearing augmentation versus stapes surgery for the treatment of otosclerosis.

Recent Findings Otosclerosis leads to reduced patient quality of life, which can be ameliorated by either stapes surgery, or hearing aid usage. The success of stapes surgery is high, and the risks of serious postoperative complications are low. Hearing aids don't have the complications of surgery but are associated with long-term costs. Cost-effectiveness models have shown that stapes surgery is a cost-effective method for treating otosclerosis.

Summary Both stapes surgery and hearing aids can improve patient-reported quality of life in otosclerosis. Stapes surgery has larger upfront costs and surgical risks, but hearing aids are associated with longer lifetime costs. Stapes surgery is costeffective for the treatment of otosclerosis.
\end{abstract}

Keywords Otosclerosis $\cdot$ Hearing aids $\cdot$ Stapedectomy $\cdot$ Stapedotomy $\cdot$ Cost-effectiveness $\cdot$ Quality of life

\section{Introduction}

Otosclerosis is an idiopathic disorder defined by abnormal bony remodeling in the otic capsule. This process leads to stapes footplate fixation, causing a progressive hearing loss that is primarily conductive in nature, but may have sensorineural components as well, especially in advanced disease. The current management paradigm for hearing loss due to otosclerosis is hearing amplification with a hearing aid or stapes surgery which can be accomplished either through stapedectomy or stapedotomy [1]. Although these are the two most common methods, there are other options. For those who are hesitant to undergo stapes surgery but struggle with traditional hearing aids the bone implanted hearing aid (BAHA) is an option [2].

This article is part of the Topical Collection on OTOLOGY:

Otosclerosis and Stapes Surgery

Danielle M. Gillard

danielle.gillard@ucsf.edu

Jeffrey D. Sharon

jeffrey.sharon@ucsf.edu

1 Department of Otolaryngology, University of California San Francisco School of Medicine, San Francisco, CA, USA
Finally, in severe otosclerosis, cochlear implantation can be considered [1].

One way to compare different interventions in medicine is to analyze their cost-effectiveness. This provided a comprehensive value comparison between treatment options, factoring in cost of complications as well. In addition to incorporating outcomes, cost-benefit analyses can also incorporate patientreported health measures and quality of life (QoL) in determining the efficacy of treatment options. Cost-effectiveness models can be helpful in analyzing surgery versus hearing aids in the treatment of otosclerosis as the success of surgery and hearing amplification by assessing cost, patient satisfaction, and quality of life. Additionally, surgery has a larger upfront cost while hearing aids are associated with a smaller but continuing cost over many years. Lastly, surgery is associated with several complications not seen with hearing aids. These individual factors can be combined in cost-effectiveness models to give a more complete picture of the relative value of each treatment strategy.

\section{Quality of Life With Otosclerosis}

The disease process of otosclerosis is known to be associated with a decreased quality of life, most commonly attributed to the symptom of hearing loss. Hearing loss is known to have 
multisystem effects on an individual, including restrictions in physical and social behaviors, and in older adults, it can influence the progression of dementia and cognitive decline [3]. Hearing loss has also been shown to be associated with a variety of psychological concerns including anxiety, stress, and depression [4]. Unilateral hearing loss, as is commonly seen in otosclerosis, has significant impacts in functionality as well, even in younger adults. Unilateral hearing loss increases difficulty with understanding speech in noise, sound localization, and detection of sound located on the side of the affected ear [5]. Unilateral hearing loss is also known to lead to psychosocial stress in these patients [6].

Hearing loss is associated with tinnitus and this is true in otosclerosis as well. In a study of 248 adults, undergoing stapedotomy for otosclerosis $64 \%$ reported chronic tinnitus [7]. Tinnitus can cause severe emotional distress for patients [8] and is an important consideration in determining patient quality of life in otosclerosis.

Although otosclerosis is commonly associated with hearing deficits, there is evidence of vestibular system involvement as well. Although vestibular system involvement is thought to be limited to advanced cases of otosclerosis that involve the bony labyrinth $[9,10]$, some papers report up to $30 \%$ of patients with otosclerosis report vestibular symptoms including unsteadiness, dizziness, and even vertiginous attacks [11]. However, this is not something we commonly see in our vestibular clinic.

The overall QoL in otosclerosis can be difficult to determine. There are a variety of different scales that assess overall QoL as well as health-related quality of life (HRQoL) that have been used in otosclerosis. Research shows that the overall health utility score for hearing loss is dependent on the measure that is chosen. For example, in a 2005 paper Barton, Bankart, and Davis[12] investigated the HRQoL in over 900 individuals with hearing loss using multiple HRQoL tools and found that, although all patients reported a significantly decreased QoL regardless of the measure that was used, all of the scores were significantly different from one another.

\section{Quality of Life After Stapes Surgery}

Stapes surgery for the treatment of otosclerosis has a high success rate in improving hearing of roughly $90 \%$ [13]. Even individuals with severe to profound hearing loss can see some improvement after stapes surgery [14]. There are two major drawbacks to stapes surgery, the first being that patients may require revision surgery, and the second is that patients may continue to have disease progression that will eventually require hearing amplification despite surgery. The rate of revision surgery is roughly between $10-20 \%$ of individuals who undergo stapes surgery [15], and $3.5 \%$ of surgical patients will have progression of hearing loss that requires amplification every year after stapes surgery [16]. There is also a $1 \%$ risk of postsurgical deafness in the treated ear [17].

Previously success in stapes surgery was based loosely on the Belfast 15/30 rule of thumb [18]. This outcome measure was based on 220 patients with hearing loss who had an improvement in hearing of at least 10 decibels $(\mathrm{dB})$ after tympanoplasty or stapes surgery. The authors of this study found that patients were likely to derive significant benefit postoperatively if the air conduction threshold in speech frequencies was less than or equal to $30 \mathrm{~dB}$ or if the interaural difference was reduced to at least less than $15 \mathrm{~dB}$. The Belfast rule of thumb that derives from this defines normal, symmetrical hearing as a hearing loss of less than $30 \mathrm{~dB}$ and an interaural difference of less than $15 \mathrm{~dB}$ at speech frequencies. Therefore, success in stapes surgery had focused on closure of the air-bone gap on the affected side. Stapes surgery has roughly $93 \%$ hearing improvement postoperatively based on audiometric data, based on the Belfast rule of thumb [19]. However, multiple studies have found that there is a lack of correlation between postoperative audiometric results and patient-reported quality of life in stapes surgery $[20,21]$.

There have been many studies assessing patient QoL after stapes surgery, mostly from Germany and Sweden. Subramaniam et al. [21] assessed a 21 patient cohort and found that $95 \%$ had a closure of air-bone gap to $\leq 20 \mathrm{~dB}$, while only $81.8 \%$ reported better postoperative QoL. Chandarana et al. found that $96 \%$ of their patient cohort reported improved QoL after stapes surgery primarily based on the Hearing Handicap Inventory (HHI) [22].

There are a variety of HRQoL scales that can be used to assess postoperative QoL. In fact, there are more than 50 different patients who reported questionnaires that are used to assess hearing loss in adults [23, 24]. There is currently one HRQoL scale that has been developed specifically for otosclerosis, the Stapesplasty Outcomes Test (SPOT-25) [25]. This scale, which is currently only validated in German, is a 5-point Likert scale that covers hearing, tinnitus, the psychological aspects of the disease, and social impairment. It was validated on 52 patients undergoing stapes surgery and was found to have a high internal consistency (Cronbach's $\alpha>0.7)$ and high test-retest reliability $(r=0.85)$. In their validation study, they found that HRQoL improved significantly after surgery. In 2018, Lailach et al. used the SPOT-25 to assess a group of 37 patients 1 day prior to stapes surgery and 3 months, postoperatively [25]. They found significant improvement in air-bone gap, bone conduction, speech recognition, and SPOT-25. However, similar to other nonspecific QoL and HRQoL questionnaires, some aspects of QoL scores correlated with degree of hearing improvement, but not all aspects did, and there was no linear degree 
of association between QoL improvement and residual hearing loss. Weiss et al. also used the SPOT-25 in 2020 to assess 30 patients who underwent stapedotomy [26]. Again, they found that, although there was a benefit in hearing outcomes, there was not a great correlation between outcome surveys and hearing performance. This survey is currently undergoing a validation study in Dutch patients [23]. This survey represents a possible future direction for assessing the HRQoL in stapes surgery if it can be validated in other languages especially since audiometric data may not be the best measurement for success from the patient perspective in stapes surgery.

As it is clear that audiometric improvement does not tell the complete story for patient-reported improvements after stapes surgery, some studies have looked to other explanations for the degree of QoL benefit. Dziendziel et al. studied the relationship of tinnitus improvement and HRQoL in stapes surgery [7]. This study found that there was a significant reduction in tinnitus after stapes surgery as measured by the Tinnitus Functional Index (TFI) [27]. A greater reduction in TFI score was associated with improved HRQoL scores and this correlation was stronger than the association with audiometric improvement based on their regression analysis. A 2018 literature review found that there is an 85\% improvement in tinnitus after stapes surgery [19]. A 2014 study found that, in patients with tinnitus prior to stapes surgery, the majority experienced improvement in tinnitus within 6 months of surgery, with the majority finding improvement within the first month. However, it is important to note that this study also found that, in individuals without prior significant tinnitus, there was a $10 \%$ risk of transient worsening tinnitus, that resolved by the 6-month mark [28]. The association between tinnitus and QoL improvement highlights that there are factors outside of hearing improvement that result in improved patient-reported outcomes after surgery.

It is also important to realize that surgery poses certain risks that are not seen with hearing aid use, which affects postoperative QoL. Surgical complications of stapes surgery are relatively rare with the rate of serious complications at about $1 \%$ [29]. These complications can either temporarily or permanently affect QoL and therefore are important considerations in analyzing cost-effectiveness of the treatment of otosclerosis.

One structure that lies in close proximity to the surgical field during stapes surgery is the chorda tympani nerve, which controls taste sensation from the ipsilateral anterior two-thirds of the tongue as well as parasympathetic salivary function of the submandibular and sublingual glands. In a study of patients with otosclerosis undergoing stapes surgery [26], preoperative taste disturbance complaints were relatively rare at $3.7 \%$. However, postoperatively reported taste disturbances were much more frequent. About $60 \%$ of patients reported taste disturbances at any time after surgery, half of the patients reported disturbances at 1 week; this number decreased to about $40 \%$ at 6 weeks and was down to $5 \%$ at 1 -year post-surgery. The most commonly reported symptoms were loss of taste or a metallic taste sensation. In this cohort of 134 patients, only one patient had documentation of transection of the chorda tympani during the surgery. From the head and neck cancer literature, it is known that taste abnormalities lead to decreased enjoyment of eating, decreased oral consumption, and reduced QoL making this an important consideration when thinking about outcomes in stapes surgery [30]. We find this to be an important consideration in our practice, especially for individuals where taste is an important aspect of their job, such as professional chefs or sommeliers.

While vestibular symptoms are not as common in otosclerosis, they are relatively common after stapes surgery. Between 12 and $50 \%$ of patients report, at least one vestibular symptom after surgery; however, these symptoms are usually mild and last less than 1 week [11]. The most commonly reported symptoms and signs are a floating sensation and rotatory vertigo [31].

Vertiginous symptoms tend to be temporary and resolve in the weeks to months after surgery. In a 2020 study, Alessandrini et al. [32] studied patients reporting vertigo after stapes surgery. They tested vestibulo-ocular reflex (VOR) gain, posturography parameters, and QoL questionnaires. They found improvement in posturographic parameters 6 weeks after stapedotomy. This was also reflected in the QoL scores, in which the patients reported better quality of life in regards to their balance function after surgery. In another study assessing patients who underwent stapes surgery, posturography was tested prior to 1 week after and 1 month after surgery [33]. This study found that patients had a reduction in balance after surgery, with their posturographic parameters returning to preoperative values after 1 month. These results were similar to a prior 1996 study by Molony and Marais that used postural sway analysis that found decreases in function after 1 week that returned to normal at retest 6 months after surgery [34]. There does appear to be a relationship between balance and postural control. In one study, patients with hearing loss had balance testing performed with and without hearing aids. Although the results were not statistically significant, there was a subset of participants that did significantly improve postural control with hearing amplification [35].

Long-term symptoms or disabling vertigo is very rare after surgery $[31,36]$. These symptoms are usually due to intraoperative complications including perilymph aspiration, perilymphatic fistula, or Benign positional paroxysmal vertigo (BPPV), likely from otolith displacement due to head positioning during surgery [11]. We have also seen in practice that patients can develop vestibular hypofunction when there is a loss of hearing after surgery. 
Severe complications such as perilymph fistula, postoperative sensorineural hearing loss, prolonged vertigo, or facial nerve injury occur at a frequency of less than $1 \%[13,37]$.

It is important to note that other surgical options for the treatment of otosclerosis are becoming more common. BAHA devices have been shown to be successful in the treatment of unilateral hearing loss, and there are studies that show that it provides a quality of life benefit in individuals with otosclerosis as well [2]. Cochlear implantation is also used in cases of advanced otosclerosis with significant sensorineural hearing loss, or in individuals who developed sensorineural hearing loss after stapes surgery. Individuals with otosclerosis and severe hearing loss show similar quality of life improvement after cochlear implantation as other users of cochlear implants with post-lingual deafness [38].

\section{Cost of Stapes Surgery}

Although there are a variety of methods to assess the effectiveness of stapes surgery, determining the actual cost of stapes surgery in the USA is more difficult. There are a variety of perspectives from which to analyze cost when building cost-effectiveness models. One could consider costs in terms of how much the hospital charges for the preoperative evaluation, surgery, anesthesia, and post-operative care. However, one could also consider direct costs to the patient themselves or even insurance reimbursement for the procedure. All methods can be valid depending on the purpose of the cost-effectiveness analysis as they all assess different aspects of our healthcare system, hospital costs, insurance costs, and patient costs. Cost-effectiveness research can focus on whether stapes surgery is cost-effective for the hospital, cost-effective for insurance providers, or cost-effective for patients. Each perspective will bring important information to the discussion of the overall cost-effectiveness of the procedure.

There are relatively few direct cost analyses of stapes surgery. In a 2003 paper by Klask and Schmelzer, they found their average cost was roughly $954 €$ for stapes surgery in Germany. They had a $6.5 \%$ complication rate with an additional 1754 and $3504 €$ to manage [39]. Since cost studies reflect the country they take place in, the value of the currency in that country, and the chronological time in which they were evaluated based on changing inflation and exchange rates, this study may not be applicable outside of Germany. Unfortunately, the majority of cost data analyses do not include an inflation-adjusted amount for comparison in other countries.

There is one published study on the cost of stapes surgery in the USA in the last decade. This 2019 study [40•] found that the average cost of stapedectomy was US $\$ 3542$ with a standard deviation (SD) of 1259 . The authors found that surgical supply and increased operative time were the most associated with increased cost, and the type of laser used to perform the stapedectomy was the supply most associated with increased cost. The strengths of this study are that it assessed average cost across more than 20 surgeons at 10 hospitals that included both tertiary-care centers and academic hospitals, as well as urban, community, and rural hospitals, all factors that can influence cost. However, the study focused on only one integrated non-profit healthcare system within the state of Utah, so it may not be as applicable to other states.

\section{Quality of Life With Hearing Aids}

Hearing aids are known to improve QoL in individuals with hearing loss. Patients without hearing aids have a worse reported QoL than patients that use them [41]. A 2018 metaanalysis of randomized clinical trials of hearing aid use in adults with mild to moderate hearing loss found that there was a beneficial effect of hearing aids on HRQoL with a smaller benefit on overall QoL [42]. In addition to improving audiometric measures, understanding of speech, and sound localization, hearing aids also improve HRQoL by reducing the psychological and emotional effects of hearing loss [43].

Hearing aids are very safe to use but are associated with some health-related complications that can affect QoL. Hearing aid use exacerbates cerumen production which can result in increased healthcare utilization for management [44]. Hearing aid use is also associated with skin allergies, otitis externa from external auditory canal obstruction, and erosive ear changes that may require surgical correction [45].

Despite their ability to improve QoL with a low side effect profile, there are other psychosocial factors that decrease hearing aid use and affect QoL. For some patients, hearing aid use can be considered an inconvenience and carries with it a perceived social stigma [46]. This is particularly important when considering hearing aids for the treatment of otosclerosis since the peak onset for otosclerosis is in the third decade of life [47] when patients are young and HRQoL is generally otherwise high.

\section{Cost of Hearing Aids}

Much like in stapes surgery, determining the cost of hearing aids can be difficult. Hearing aids are widely variable in cost. The average hearing aid cost in the USA is between US $\$ 1000$ and 4000 in the year 2020. The cheapest hearing aids cost between US $\$ 500$ and 3000 while premium models cost about US \$4500-6000 per device [48•]. Higher quality hearing aids can last many years, but basic hearing aids require replacement about every 4 years [49]. Additionally, hearing aids and hearing aid fittings require frequent audiology 
appointments to ensure proper programming which adds additional cost. As patients with otosclerosis can present relatively early in their adult life they often have to bear many years of cost for hearing aid upkeep.

Traditionally, most adult patients in the USA had to pay out of pocket for hearing aids which placed the cost perspective entirely on the payee. Thus, the hospital and insurance perspectives only reflect the cost of audiology appointments and medical complications of hearing aid maintenance. The Federal Drug Authority (FDA) reorganization act of 2017 [50] includes the Over the Counter Hearing Aid Act which creates a new category of over-the-counter hearing aid devices to improve hearing in adults with mild to moderate hearing impairment. This poses the possibility for large changes in the affordability and accessibility of hearing aids in the future. One major exception to this rule is the Veterans Health Administration which covers the cost of hearing aids, repairs, and future batteries.

\section{Cost-Effectiveness of Hearing Aids Versus Stapes Surgery in the Treatment of Otosclerosis}

There is a dearth of research directly comparing the costeffectiveness of stapes surgery versus hearing aids for the treatment of otosclerosis. In a 2009, paper Savvas and Maurer [51] compared the cost of stapes surgery versus the average cost of lifetime hearing aid use in Germany. They found that over a lifetime hearing aid cost was greater than the cost of stapedectomy even with a 5-10\% revision rate and with $20 \%$ of patients going on to require hearing aids despite surgery. Their costs covered the costs of surgery, anesthesia, 3-5-day hospital stay (if needed for postoperative complications or concerns), postoperative care, and audiology evaluation based on 164 stapedotomy cases. Surgery remained cheaper than hearing aids at three different theoretical ages 30,50 , and 60 years of age.

It is important to note the differences in healthcare delivery systems between Germany and the USA. Germany has a universal multi-payer system in which there is a combination of national healthcare insurance plans and private insurance plans [52], while the USA functions on a convoluted mix of different types of public and private insurance-based plans which means that these results may not have high external validity for countries with different healthcare systems.

There is only one paper directly comparing the cost versus quality of life benefit between stapes surgery and hearing aids in otosclerosis that is currently published [53••]. In this study, a Markov model [54] was used to model multi-year outcomes and compare the lifetime costs of stapedectomy versus hearing aids in regards to QoL. A Markov model is a stochastic model that models multi-year outcomes using the probability of progression from one state to another based on a patients' current state. The outcome in this study was the incremental cost-effectiveness (ICER) of stapedectomy, which is defined as the difference in lifetime costs divided by the difference in lifetime QoL benefit. Costs were measured based on Medicare national mean payments for stapes surgery in the USA and the average initial cost of hearing aids and yearly maintenance costs. The effectiveness was calculated using quality of life years (QALY) in which zero equates to death and 1 signifies 1 year of so-called perfect health. This study also factored in the probability of revision surgery and the probability of progression to hearing aid use despite surgery. This study found that stapedectomy cost about US $\$ 3000$ more than hearing aids over a lifetime and had an incremental effectiveness of about 1 QALY. Thus the ICER ratio for stapedectomy was about US $\$ 4000$. In costeffectiveness research, an ICER of US $\$<50,000$ is considered cost-effective [55]. Thus, the authors of this paper concluded that stapedectomy was a cost-effective method for the treatment of otosclerosis in the USA. It is important to note that this study did not include the costs associated with the progression to the need for BAHA or cochlear implantation.

Additional research in cost-effectiveness using data from actual hospital costs at different institutions and actual costs to patients is needed to determine a more accurate picture for the cost-effectiveness of these treatment paradigms in the USA. It is also important to note that the US Food and Drug Administration (FDA) Reauthorization Act of 2017 will make certain hearing aids available over the counter at a lower cost for those with mild to moderate hearing loss which may change cost-effectiveness models in years to come.

\section{Conclusions}

Otosclerosis decreases patient QoL, and patients can get significant improvement with either hearing aids or stapes surgery. Stapes surgery is effective in improving hearing based on audiometric parameters as well as patient HRQoL surveys. Stapes surgery is safe with a low rate of serious complications but can be associated with the need for repeat surgery or continued progression of disease. The cost of stapes surgery can be viewed based on the perspective of hospitals, patients, or insurers and can be drastically different across different locations. Hearing aids improve QoL with limited medical side effects but are associated with a high out-of-pocket cost to the consumer. They also carry additional social stigma, especially for young users. There is a lack of research comparing direct cost-effectiveness of these two treatment regimens, but the two papers currently published support that stapedectomy is a cost-effective method for the treatment of otosclerosis compared to hearing aids. 


\section{Compliance with Ethical Standards}

Conflict of Interest Danielle Marie Gillard and Jeffrey D Sharon declare that they have no conflict of interest.

Human and Animal Rights and Informed Consent This article does not contain any studies with human or animal subjects performed by any of the authors.

Open Access This article is licensed under a Creative Commons Attribution 4.0 International License, which permits use, sharing, adaptation, distribution and reproduction in any medium or format, as long as you give appropriate credit to the original author(s) and the source, provide a link to the Creative Commons licence, and indicate if changes were made. The images or other third party material in this article are included in the article's Creative Commons licence, unless indicated otherwise in a credit line to the material. If material is not included in the article's Creative Commons licence and your intended use is not permitted by statutory regulation or exceeds the permitted use, you will need to obtain permission directly from the copyright holder. To view a copy of this licence, visit http://creativecommons.org/licenses/by/4.0/.

\section{References}

Papers of particular interest, published recently, have been highlighted as:

- Of importance

• Of major importance

1. Berrettini S, Burdo S, Forli F, Ravecca F, Marcaccini M, Pietro Casani A, et al. Far Advanced otosclerosis: stapes surgery or cochlear implantation? Journal Otolaryngol. 2004. p. 165. https://doi.org/ 10.2310/7070.2004.03006

2. McLarnon CM, Davison T, Johnson IJM. Bone-anchored hearing aid: comparison of benefit by patient subgroups. Laryngoscope. 2004;114:942-4.

3. Arlinger S. Negative consequences of uncorrected hearing lossa review. Int J Audiol. 2003. pp. 17-20. https://doi.org/10.3109/ 14992020309074639

4. Jafari Z, Kolb BE, Mohajerani MH. Age-related hearing loss and tinnitus, dementia risk, and auditory amplification outcomes. Ageing Res Rev. 2019;56: 100963.

5. McLeod B, Upfold L, Taylor A. Self reported hearing difficulties following excision of vestibular schwannoma. Int J Audiol. 2008;47:420-30.

6. Giolas TG, Wark DJ. Communication problems associated with unilateral hearing loss. J Speech Hear Disord. 1967;32:336-43.

7. Dziendziel B, Skarzynski H, Gos E, Skarzynski PH. Changes in hearing threshold and tinnitus severity after stapes surgery: which is more important to the patient's quality of life? ORL. 2019. pp. 224-233. https://doi.org/10.1159/000500992

8. Mazurek B, Boecking B, Brueggemann P. Association between stress and tinnitus-new aspects. Otol Neurotol. 2019;40:e467-73.

9. Hayashi H, Cureoglu S, Schachern PA, Oktay MF, Fukushima H, Sone M, et al. Association between cupular deposits and otosclerosis. Arch Otolaryngol Head Neck Surg. 2006. p. 1331. https:// doi.org/10.1001/archotol.132.12.1331

10. Bertholon P, Karkas A. Otologic disorders causing dizziness, including surgery for vestibular disorders. Handb Clin Neurol. 2016;137:279-93.
11. Kujala J, Aalto H, Hirvonen T. Video-oculography findings and vestibular symptoms on the day of stapes surgery. Eur Arch Otorhinolaryngol. 2010;267:187-90.

12. Barton GR, Bankart J, Davis AC. A comparison of the quality of life of hearing-impaired people as estimated by three different utility measures. Int J Audiol. 2005;44:157-63.

13. Vincent R, Sperling NM, Oates J, Jindal M. Surgical findings and long-term hearing results in 3,050 stapedotomies for primary otosclerosis: a prospective study with the otology-neurotology database. Otol Neurotol. 2006;27:S25-47.

14. Caylakli F, Yavuz H, Yilmazer C, Yilmaz I, Ozluoglu LN. Effect of preoperative hearing level on success of stapes surgery. Otolaryngol Head Neck Surg. 2009;141:12-5.

15. Lippy WH, Wingate J, Burkey JM, Rizer FM, Schuring AG. Stapedectomy revision in elderly patients. The Laryngoscope. 2002. pp. 1100-1103. https://doi.org/10.1097/00005537-200206000-00030

16. Redfors YD, Möller C. Otosclerosis: thirty-year follow-up after surgery. Ann Otol Rhinol Laryngol. 2011;120:608-14.

17. Martin MBS, St. Martin MB, Rubinstein EN, Hirsch BE. High-frequency sensorineural hearing loss after stapedectomy. Otol Neurotol. 2008. pp. 447-452. https://doi.org/10. 1097/mao.0b013e318172d6a3

18. Smyth GD, Patterson CC. Results of middle ear reconstruction: do patients and surgeons agree? Am J Otol. 1985;6:276-9.

19. Cavalcante AMG, Silva IM de C, Neves BJ, Oliveira CA, Bahmad F Jr. Degree of tinnitus improvement with stapes surgery - a review. Braz J Otorhinolaryngol. 2018;84: 514-518.

20. Redfors YD, Olaison S, Karlsson J, Hellgren J, Möller C. Hearingrelated, health-related quality of life in patients who have undergone otosclerosis surgery: a long-term follow-up study. Int J Audiol. 2015;54:63-9.

21. Subramaniam K, Eikelboom RH, Marino R, M. D. Atlas, Rajan GP. Patient's quality of life and hearing outcomes after stapes surgery. Clin Otolaryngol. 2006. pp. 273-279. https://doi.org/ 10.1111/j.1749-4486.2006.01237.x

22. Chandarana S, Parnes L, Agrawal S, Fung K. Quality of life following small fenestra stapedotomy. Ann Otol Rhinol Laryngol. 2005; 114:472-7.

23. Blijleven EE, Thomeer HGXM, Stokroos R, Wegner I. Protocol for a validation study of the translated stapesplasty outcome test 25 for measurement of disease-specific quality of life in Dutch patients with otosclerosis. BMJ Open. 2019;9: e030219.

24. Granberg S, Dahlström J, Möller C, Kähäri K, Danermark B. The ICF Core Sets for hearing loss--researcher perspective. Part I: systematic review of outcome measures identified in audiological research. Int J Audiol. 2014;53: 65-76.

25. Lailach S, Schenke T, Baumann I, Walter H, Praetorius M, Beleites T, et al. Living with otosclerosis: disease-specific health-related quality-of-life measurement in patients undergoing stapes surgery. Eur Arch Otorhinolaryngol. 2018;275:71-9.

26. Berling Holm K, Knutsson J, Strömbäck K, Danckwardt Lillieström N, Papatziamos G, Rosenblad A, et al. Taste disturbance after stapes surgery: an evaluation of frequency, severity, duration, and quality-of-life. Acta Otolaryngol. 2017;137:39-43.

27. Meikle MB, Henry JA, Griest SE, Stewart BJ, Abrams HB, McArdle $\mathrm{R}$, et al. The tinnitus functional index: development of a new clinical measure for chronic, intrusive tinnitus. Ear Hear. 2012;33:153-76.

28. Chang CYJ, Cheung SW. Tinnitus modulation by stapedectomy. Otol Neurotol. 2014;35:1065-9.

29. Antonelli PJ. Prevention and management of complications in otosclerosis surgery. Otolaryngol Clin North Am. 2018;51:453-62.

30. Hutton JL, Baracos VE, Wismer WV. Chemosensory dysfunction is a primary factor in the evolution of declining nutritional status and quality of life in patients with advanced cancer. J Pain Symptom Manage. 2007;33:156-65. 
31. Catalano N, Cammaroto G, Galletti B, Freni F, Nicita RA, Azielli $\mathrm{C}$, et al. The role of cVEMPs and vHIT in the evaluation of otosclerosis and its eventual vestibular impairment: preliminary findings. B-ENT. 2017;13:31-6.

32. Alessandrini M, Viziano A, Roselli L, Micarelli A. Surgical treatment of otosclerosis leading to changes in postural control and quality of life. Laryngoscope. 2020;130:2448-54.

33. Ozmen AO, Aksoy S, Ozmen S, Saraç S, Sennaroğlu L, Gürsel B. Balance after stapedotomy: analysis of balance with computerized dynamic posturography. Clin Otolaryngol. 2009;34:212-7.

34. Molony NC, Marais J. Balance after stapedectomy: the measurement of spontaneous sway by posturography. Clin Otolaryngol. 1996. pp. 353-356. https://doi.org/10.1111/j.1365-2273.1996. tb01086.x

35. Weaver TS, Shayman CS, Hullar TE. The effect of hearing aids and cochlear implants on balance during gait. Otol Neurotol. 2017;38:1327-32.

36. Albera R, Canale A, Lacilla M, Cavalot AL, Ferrero V. Delayed vertigo after stapes surgery. Laryngoscope. 2004;114:860-2.

37. Brookler KH. Otosclerosis surgery: reassessment of its value in 1978. Laryngoscope. 1979;89:725-9.

38. Calvino M, Sánchez-Cuadrado I, Gavilán J, Lassaletta L. Cochlear implant users with otosclerosis: are hearing and quality of life outcomes worse than in cochlear implant users without otosclerosis? Audiol Neurootol. 2018;23:345-55.

39. Klask J. Schmelzer A [Postoperative complications in stapes surgery. An analysis of medical and economic aspects]. HNO. 2003;51:893-7.

40. Casazza GC, Thomas AJ, Dewey J, Gurgel RK, Shelton C, Meier JD. Variations in stapes surgery cost within a multihospital network. Otolaryngol Head Neck Surg. 2019;161: 835-841. This article reflects average cost of stapedectomy in the USA, looking at average costs within one hospital system across different surgeons.

41. Hyams AV, Hay-McCutcheon M, Scogin F. Hearing and quality of life in older adults. J Clin Psychol. 2018;74:1874-83.

42. Brennan-Jones CG, Weeda E, Ferguson M. Cochrane corner: hearing aids for mild to moderate hearing loss in adults. Int $\mathbf{J}$ Audiol. 2018;57:479-82.

43. Chisolm TH, Johnson CE, Danhauer JL, Portz LJP, Abrams HB, Lesner S, et al. A systematic review of health-related quality of life and hearing aids: final report of the American Academy of Audiology Task Force On the Health-Related Quality of Life Benefits of Amplification in Adults. J Am Acad Audiol. 2007;18:151-83.

44. Roeser RJ, Ballachanda BB. Physiology, pathophysiology, and anthropology/epidemiology of human earcanal secretions. J Am Acad Audiol. 1997;8:391-400.
45. Owen HH, Rosborg J, Gaihede M. Cholesteatoma of the external ear canal: etiological factors, symptoms and clinical findings in a series of 48 cases. BMC ear, nose and throat disorders. 2006. https://doi.org/10.1186/1472-6815-6-16

46. Mohr PE, Feldman JJ, Dunbar JL. The societal costs of severe to profound hearing loss in the United States. Policy Anal Brief H Ser. 2000;2:1-4.

47. Otosclerosis LA. A genetic and clinical study. Acta Otolaryngol Suppl. 1960;154:1-86.

48. Rains T. How Much Do Hearing Aids Cost? (2021). ConsumerAffairs; 4 May 2021 [cited 14 Aug 2021]. Available: https:// www.consumeraffairs.com/health/hearing-aid-cost.html. This paper reflects the current costs for hearing aids in the USA including average device costs and maintenance costs.

49. Committee on accessible and affordable hearing health care for adults, board on health sciences policy, health and medicine division, national academies of sciences, engineering, and medicine. Hearing health care for adults: priorities for improving access and affordability. Blazer DG, Domnitz S, Liverman CT, editors. Washington (DC): National Academies Press (US); 2016.

50. United States. Congress. House. Committee on energy and commerce. FDA Reauthorization Act of 2017: report (to accompany H.R. 2430). 2017.

51. Savvas E, Maurer J. Economic viability of stapes surgery in Germany. J Laryngol Otol. 2009;123:403-6.

52. Bärnighausen T, Sauerborn R. One hundred and eighteen years of the German health insurance system: are there any lessons for middle- and low-income countries? Soc Sci Med. 2002. pp. 1559-1587. https://doi.org/10.1016/s0277-9536(01)00137-x

53.• Gillard DM, Harris JP. Cost-effectiveness of stapedectomy vs hearing aids in the treatment of otosclerosis. JAMA Otolaryngol Head Neck Surg. 2020;146: 42-48. This is the only paper that compares the direct cost-effectiveness between hearing aids and stapedectomy for the treatment of otosclerosis. It found that surgery is a cost-effective method for the treatment of otosclerosis.

54. Hasselblad V, McCrory DC. Meta-analytic tools for medical decision making: a practical guide. Med Decis Mak. 1995. pp. 81-96. https://doi.org/10.1177/0272989x9501500112

55. Grosse SD. Assessing cost-effectiveness in healthcare: history of the $\$ 50,000$ per QALY threshold. Expert Rev Pharmacoecon Outcomes Res. 2008;8:165-78.

Publisher's Note Springer Nature remains neutral with regard to jurisdictional claims in published maps and institutional affiliations. 